\title{
DA LUDOTERAPIA NÃO-DIRETIVA À LUDOTERAPIA CENTRADA NA CRIANÇA -DESENVOLVIMENTO HISTÓRICO
}

\author{
From non-directive play therapy to child-centered play therapy - historical development
}

De la terapia de juego no-directiva a terapia de juego centrada en el nino - desarollo histórico

Rosa Angela Cortez de Brito

José CÉLIO FREIRE

Lucas Guimarães Bloc

Virginia de Saboia Moreira Cavalcanti

\begin{abstract}
Resumo: O desenvolvimento da Abordagem Centrada na Pessoa foi dividido em fases que apresentam as atitudes que fazem parte da relação terapêutica, independente do público atendido. Contudo, na especificidade da psicoterapia infantil, o desenvolvimento teórico e prático da própria abordagem aponta para a existência de peculiaridades. Este estudo tem como objetivo apresentar, através de uma revisão narrativa de literatura, fundamentada na hermenêutica filosófica de Gadamer, as possíveis características relativas ao atendimento de crianças a partir de um retorno às fases da ACP. Discutimos os fundamentos iniciais do atendimento com crianças em Rogers ena perspectiva clássica de Axline, compreendendo como a ludoterapia com crianças se caracteriza nas fases não-diretiva, reflexiva, experiencial e pós-rogeriana. Discutimos sobre propostas contemporâneas nos contextos europeu, estadunidense e brasileiro. Concluímos que essa delimitação do atendimento infantil possibilita uma percepção mais clara das mudanças e aprimoramentos em relação aos trabalhos pioneiros de Rogers e Axline, sempre tendo como eixo a dimensão relacional e a tendência ao crescimento como motor do processo terapêutico.
\end{abstract}

Palavras-chave: Psicologia humanista; Terapia centrada no cliente; Psicoterapia da criança; Ludoterapia.

\begin{abstract}
The development of the Person-Centered Approach was divided into phases that presents the attitudes that are part of therapeutic relationship, regardless of the public served. However, in the specificity of child psychotherapy, the theoretical and practical development of the approach points to the existence of peculiarities. This study aims to present, through narrative literature review, based on Gadamer's philosophical hermeneutics, the possible characteristics related to the child therapy, starting from a return to the PCA phases. We discuss the foundations of child therapy in Rogers and Axline's classic perspective, understanding how child play therapy is characterized in non-directive, reflective, experiential and post-Rogerian phases. We discuss contemporary proposals in the European, American, and Brazilian contexts. We conclude that this delimitation enables a clearer perception of changes and improvements amongst to the Rogers and Axline's pioneering work, always having as its axis the relational dimension and the tendency to growth as a motor of the therapeutic process.
\end{abstract}

Keywords: Humanistic psychology; Client centered therapy; Child psychotherapy; Play therapy.

Resumen: El desarrollo del Enfoque Centrado en la Persona se dividió en fases que presentan las actitudes de la relación terapéutica, independiente del público. Sin embargo, en la especificidad de la psicoterapia infantil, el desarrollo teórico y práctico del enfoque en sí mismo apunta la existencia de peculiaridades. Este estudio tiene como objetivo presentar, a través de una revisión narrativa de la literatura, basado em la hermenéutica filosófica de Gadamer, las posibles características de la psicoterapia con niños, desde el retorno a las fases de ECP. Discutimos los fundamentos de la psicoterapia con niños en Rogers, la perspectiva clásica de Axline, comprendiendo como la terapia de juego com los niños se caracterizan em las fases no directiva, reflexiva, experiencial y pos-rogeriana. Discutimos propuestas contemporáneas en los contextos europeo, estadounidense y brasileño. Concluimos que esta delimitación permite una percepción más clara de los cambios y mejoras en relación con los trabajos pioneros de Rogers y Axline, teniendo siempre como eje la dimensión relacional y la tendencia al crecimiento como motor del proceso terapéutico.

Palabras clave: Psicologia humanista; Terapia centrada en el cliente; Psicoterapia del niño; Terapia de juego. 


\section{Introdução}

O desenvolvimento da teoria e prática da Abordagem Centrada na Pessoa (ACP) foi dividido em fases por comentadores do pensamento de Carl Rogers (Hart \& Tomlinson, 1970; Puente, 1970; Wood, 1983; Huizinga, 1984; Segrera, 2002; Moreira, 2010) na tentativa de contemplar elementos epistemológicos e práticos distintos que impactaram ao longo dos anos aatuação nessa perspectiva (Branco, 2010; Moreira, 2010). Os estudos sobre a sistematização por fases na ACP não se propõem a apresentar delimitações relativas à faixa etária, ao gênero, dentre outros, mas sim acerca das atitudes na atuação que devem fazer parte de toda relação terapêutica, independente do público atendido. No caso da psicoterapia com crianças na ACP, a faixa etária é um sinalizador para uma prática que possui características próprias. O modo como a criança é reconhecida, a comunicação das atitudes no acontecimento do atendimento, assim como a maneira como as intervenções se estabelecem passam a apresentar especificidades quando o público atendido é o infantil. Esses contornos específicos têm implicação direta no modo como a teoria e prática da psicoterapia com crianças é apresentada, proposta e desenvolvida.

No contexto específico da psicoterapia com crianças, o desenvolvimento teórico e prático da ACP aponta para a existência de peculiaridades (VanFleet, Sywulak, \& Sniscak, 2010; Landreth, 2012; Axline, 2013/1947; Bratton, Purswell \& Jayne, 2015) e que estas existem com certa estabilidade, a despeito da forma como a proposição teórica é apresentada. O próprio Rogers (1942/2005) delimita, de forma breve, elementos que seriam particulares do atendimento psicoterápico infantil, tais como: a relação, que se daria mais através das ações do que das palavras; a natureza da ligação entre a criança e a família como elemento que atravessa a psicoterapia; a relevância do acompanhamento conjunto dos pais e da criança, com psicólogos diferentes. Se na psicoterapia de adultos, o estabelecimento da relação terapêutica se dá, via de regra, pela comunicação verbal, com a criança, a brincadeira é a via de comunicação e reconhecimento de suas experiências (Döring, 2008; Landreth, 2012; Axline, 2013/1947). Outro aspecto peculiar e fundamental da psicoterapia com crianças diz respeito à relevância da participação da família no desenvolvimento do processo terapêutico (Hillman, 2018; Perryman \& Bowers, 2018).

Considerando que a divisão da ACP por fases tem sido um recurso relevante para sua compreensão histórica, nos questionamos como se daria o processo de desenvolvimento histórico da Ludoterapia Centrada na Criança em cada uma dessas fases. Em pesquisas recentes sobre a Ludoterapia Centrada na Criança, verificamos que o reconhecimento das particularidades da psicoterapia com crianças tem sido destacado, em diversos países (Bratton, Purswell \& Jayne, 2015; Lin \& Bratton, 2014) e também em contexto brasileiro (Branco, 2001; Andrade \& Cavalcante Júnior, 2008; Vasconcelos, 2008; Campos \& Cury, 2009; Brito \& Freire, 2014; Brito \& Paiva, 2012), a partir de estudos de natureza teórica e empírica.

Os estudos que discutem a Ludoterapia Centrada na Criança tomam como ponto de partida, via de regra, a ludoterapia não-diretiva proposta por Virginia Mae Axline (2013/1947) e buscam compreender a dimensão prática da psicoterapia com crianças como forma de ampliar e aprofundar a proposta clássica de Axline (Moustakas, 1953; 2007; VanFleet, Sywulak, \& Sniscak, 2010; Landreth, 2012). Outros aprimoramentos dessa teoria, todavia, não mantêm como base teórica apenas os trabalhos de Axline, mas se organizam apresentando novas leituras, que também são denominadas de Ludoterapia Centrada na Criança e dialogam com outras perspectivas como, por exemplo, a saúde mental (Baggerly, Ray \& Bratton, 2010), a filosofia (Brito \& Freire, 2014) e ainda intervenções com públicos específicos (Silva \& Barroso, 2017).

Apesar dos aprimoramentos disponíveis na literatura, não verificamos estudos que contemplem uma sistematização histórica de como a teoria e prática em Ludoterapia Centrada na Criança tem se desenvolvido. Para contribuir com esse contexto, este estudo objetiva apresentar, a partir de um retorno às fases da Abordagem Centrada na Pessoa, possíveis características relativas ao atendimento de crianças. Compreendemos que essa discussão possibilita tanto o acesso à evolução histórica da Ludoterapia Centrada na Criança, como também à abertura de espaço para maior reflexão acerca da fundamentação teórica e das formas de atuação nessa modalidade de psicoterapia.

Este trabalho se constitui como uma pesquisa de revisão narrativa (Elias, Silva, Martins, Ramos, Souza \& Hipólito, 2012), que visa descrever e discutir, a partir de uma contextualização ou de um aporte teórico, o estado da arte de um determinado tema. A fundamentação filosófica dessa revisão inspirase na hermenêutica filosófica de Gadamer (2008). No contexto científico, a hermenêutica filosófica possibilita o reconhecimento de convergências entre as obras estudadas, o que abre espaço para o estabelecimento de novas verdades que apresentam importância histórica. A partir do “diálogo” entre dois horizontes, o do leitor e o do autor, pode ocorrer a proposição de um novo horizonte compreensivo do fenômeno investigado (Gadamer, 2008).

Para o desenvolvimento deste artigo buscamos estudos que apresentassem em seu resumo, palavraschave ou no corpo do texto o descritor Ludoterapia Centrada na Criança, nos idiomas português, inglês e espanhol. A busca foi realizada em teses, dissertações, livros e artigos científicos, sem demarcação de data de publicação. A seguir, tomando como base inicial as 
fases da ACP discutidas por Segrera (2002) e Moreira (2010), realizamos a leitura, a contextualização e a discussão crítica dos textos de acordo com as características da psicoterapia apresentadas em cada fase da abordagem, buscando uma inteligibilidade e sistematização para o desenvolvimento histórico da Ludoterapia Centrada na Criança.

A discussão desse artigo se dará através do desenvolvimento da teoria e da prática da Ludoterapia tanto na construção de seus fundamentos iniciais quanto em cada uma das fases da Abordagem Centrada na Pessoa. Destacamos ainda que nos deteremos às três fases da teoria referentes à psicoterapia - não-diretiva, reflexiva e experiencial -, bem como a fase pós-rogeriana, apontadas por Segrera (2002) e Moreira (2010). Como a finalidade desse estudo tem relação direta com a psicoterapia, optamos por não trabalhar com a fase coletiva/interhumana pelo fato de Rogers ter se voltado para contextos diferentes do psicoterápico (Moreira, 2010).

\section{Fundamentos Iniciais do Atendimento de Crianças na ACP (1939-1942)}

Rogers iniciou o trabalho clínico com crianças no Institute for Child Guidance, realizando psicodiagnóstico e atendimento de crianças, bem como orientação de pais. Pela impossibilidade de trabalho com a psicoterapia, atuação à época restrita ao campo da Psiquiatria, Rogers atendia no modelo de aconselhamento psicológico, no qual realizava as intervenções clínicas (Rogers, 1961/2001). Em seguida, trabalhou na Society for the Prevention of Cruelty to Children, onde conduzia psicodiagnósticos com base no referencial psicanalítico (Kirschenbaum, 2007). A visão crítica de Rogers acerca da psicanálise que ele praticava e o contato com autores neofreudianos (Branco, Vieira, Cirino, \& Moreira, 2016) fomentaram a perspectiva apresentada em $O$ Tratamento Clínico da Criança Problema (Rogers, 1939/1978). Nesta obra, Rogers discute a avaliação e o tratamento de crianças com problemas comportamentais e ressalta possibilidades de compreensão da criança, de seu comportamento e do tratamento, bem como aponta elementos para o acompanhamento com a família e a escola.

Reconhecemos em certos aspectos apresentados $O$ Tratamento Clínico da Criança Problema, mesmo que de forma inicial, algumas noções que influenciarão a Ludoterapia Centrada na Criança, a saber: a importância de enfatizar a criança ao invés do sintoma comportamental que ela apresenta; a terapia com o objetivo de ajudar a criança a desenvolver-se, para promoção de maior independência e responsabilidade. $\mathrm{O}$ autor ressalta ainda a importância das atitudes familiares, da escola e de outros espaços relevantes na vida da criança. Em obra posterior, Rogers (1942/2005) discutirá que estes contextos têm implicação direta para o sucesso ou fracasso do acompanhamento psicológico da criança.

Para Rogers (1939/1978), o ser humano é entendido como um organismo com potencial positivo de desenvolvimento e com necessidades que vão além do fisiológico: a necessidade de resposta afetiva e de reconhecimento e a necessidade de satisfação. Essa compreensão inicial acerca do organismo, de seu potencial e das necessidades que lhe são inerentes podem ser entendidos, segundo Telles, Boris, \& Moreira (2014), como aporte inicial da noção de tendência atualizante, que é fundamento da ACP (Rogers, 2005/1980).

Também verificamos que Rogers (1939/1978) preconiza que a criança precisa ser vista para além dos problemas de ordem comportamental: "abordaremos a criança, e não os sintomas comportamentais [...] existem crianças - meninos e meninas - com backgrounds e personalidades diferentes [...] é com a criança que devemos lidar, não com a generalização que fazemos a seu respeito de seu comportamento" (p. 20-21). Compreendemos que essa forma de reconhecimento da criança retira o foco no sintoma e no problema e propõe um acesso à criança tal como ela se apresenta no contato com a psicóloga, o que parece se apresentar como uma perspectiva diferente dos modelos vigentes à época de publicação da obra.

A ênfase na criança nos remete ainda à atitude de consideração positiva incondicional, descrita por Rogers (1957/1994), como uma experiência que "implica em uma forma de apreciar o cliente como uma pessoa individualizada, a quem se permite ter os próprios sentimentos, suas próprias experiências" (p. 163). Essa atitude será mencionada apenas futuramente na obra rogeriana, como condição necessária e suficiente para a mudança, a partir do processo de psicoterapia (Rogers, 1957/1994; Kinget \& Rogers, 1962/1977).

As alterações comportamentais decorrentes do atendimento psicológico dependeriam, para Rogers (1939/1978), mais da qualidade interacional da profissional e da criança do que das técnicas realizadas. Essa interação depende de qualificações que são descritas como necessárias para o sucesso do tratamento: a capacidade da psicoterapeuta de demostrar respeito e aceitação pela criança, ao invés das atitudes de frieza, impessoalidade ou de julgamento, o respeito pelo indivíduo, a compreensão da terapeuta de si mesma e dos seus padrões emocionais e, por último, os conhecimentos de Psicologia (Rogers,1939/1978).

O Tratamento Clínico da Criança Problema costuma ser citado na literatura por sua importância histórica, ora como ponto inicial do trabalho clínico de Rogers, ora como obra anterior ao desenvolvimento 
da Abordagem Centrada na Pessoa (Sollod, 1978; Moreira, 2010; Branco, Vieira, Cirino, \& Moreira, 2016). Além disso, consideramos relevante citá-la pela importância dos apontamentos iniciais acerca do acompanhamento com crianças e sua influência no desenvolvimento posterior da prática clínica. Compreendemos que as contribuições do trabalho clínico com crianças apresentadas por Rogers (1939/1978) são um ponto de partida fundamental na ACP em que são cunhados importantes fundamentos não apenas para a psicoterapia com crianças, mas para a abordagem como um todo.

\section{A Ludoterapia na Fase Não-Diretiva (1940-1950)}

\section{A Ludoterapia Não-Diretiva de Rogers}

Na obra Psicoterapia e Consulta Psicológica, de 1942, Rogers compreendia a consulta psicológica como facilitadora de uma maior independência e integração do indivíduo. A terapia não seria uma preparação para a mudança, mas sim a própria mudança (Rogers, 1942/2005; Melo, Lima, \& Moreira, 2014). A Ludoterapia é descrita no mesmo livro como uma terapia de natureza expressiva, que se desenvolve mais através das ações do que de palavras. Apesar de destacar essa diferença, Rogers compreendia o atendimento de crianças como similar à consulta psicológica do adulto:

Embora a ludoterapia possa parecer a alguns uma experiência de um tipo muito diferente da consulta psicológica de estudantes ou da terapia com pais e adultos, as suas estruturas revelam-se muito semelhantes (...). A diferença mais notável está em que na ludoterapia a relação é definida muito mais através de ações do que das palavras. (Rogers, 1942/2005, p. 94-95)

Nesse interim, Rogers (1942/2005) buscou formular critérios indicativos da necessidade de terapia, tanto para os pais quanto para a criança. O acompanhamento seria aconselhável quando as seguintes condições ocorressem: os problemas da criança tivessem relação com a interação familiar; a criança não fosse afetiva e espacialmente independente de sua família; os pais ou a criança percebessem a necessidade de apoio, o que criaria o contexto para o acompanhamento terapêutico.

Os pais seriam considerados "tratáveis" (grifo no original) caso apresentassem: a) satisfação em suas relações sociais e conjugais; b) estabilidade (Rogers não desenvolve essa noção em seu texto); c) nível intelectual médio ou superior; d) certa juventude para se adaptar a situações novas. A criança era considerada "tratável” (grifo no original) se apresentasse: a) estabilidade fisiológica; b) nível intelectual médio ou superior; c) idade suficiente para expressar-se através dos materiais e jogos. Caso fosse necessário o acompanhamento conjunto para os pais e a criança, a ludoterapia com a criança e a escuta dos pais deveria ocorrer separadamente e com profissionais diferentes (Rogers, 1942/2005).

Esses posicionamentos nos apontam uma tentativa de formular uma metodologia da prática de atendimentos com a família e com a criança, buscando garantir a eficácia da escuta psicológica. Nesse período de sua teoria, Rogers (1942/2005) ressaltava a importância da busca de uma eficácia dos atendimentos. Nessa busca, apontou também possíveis fatores que, segundo o autor, poderiam contribuir para o fracasso da psicoterapia com o público infantil: a dependência afetiva da criança em relação a seus pais; a sujeição dela ao controle familiar; a permanência da criança no lar, no decorrer do acompanhamento psicológico. Além disso, ele considera que a psicoterapia considerada eficaz implicaria necessariamente no tratamento conjunto dos pais. A terapia realizada somente com a criança poderia levá-la a uma posição de radicalidade diante dos pais, o que contribuiria, segundo o autor, para o agravamento do problema. Os pais poderiam também se tornar ciumentos por causa da relação estabelecida entre a terapeuta e a criança.

Como elementos característicos dos atendimentos com crianças discutidos por Rogers nesse período, apontamos a ênfase em um tratamento psicológico eficaz, a busca de critérios para o tratamento da criança e dos pais e dos fatores que conduziriam ao sucesso ou ao fracasso dos atendimentos. $\mathrm{Na}$ medida em que a criança era vista como alguém dependente da família, o sucesso da psicoterapia estaria diretamente ligado à psicoterapeuta e também à participação da família no tratamento. Vale ressaltar que o posicionamento que Rogers assume possibilita um olhar mais amplo para a psicoterapia infantil, através da descentralização do problema e do entendimento da criança que se apresenta como participante ativo na consulta psicológica.

\section{A Ludoterapia Autodiretiva de Axline}

Virginia Mae Axline, aluna e colaboradora de Rogers, publicou em Ludoterapia-Adinâmicainterior da infância, de 1947, os elementos característicos deste tópico. Apesar de influenciada pelo referencial não-diretivo rogeriano, Axline afirma que o termo “auto-diretiva” (Axline, 1947/2013, p.26) seria mais condizente para caracterizar sua prática do que o termo “não-diretiva”. Para Axline (1947/2013), a brincadeira é o meio natural de expressão da criança, que comunica e expressa seus sentimentos através dos brinquedos.

Em Ludoterapia - A dinâmica interior da infância, Axline apresenta sua compreensão de personalidadepela noção de ajustamento da criança. Seu pressuposto central é uma poderosa 
força, existente em cada indivíduo, direcionada para uma completa autorrealização, maturidade, independência e autodireção. Estes são parte do crescimento da criança, considerado dinâmico e marcado pelas experiências ao longo de sua vida. Essa caracterização se assemelha à noção de tendência atualizante, que será apontada por Rogers em momento posterior de sua obra (Rogers, 1951/1992), na fase reflexiva. Para Axline (1947/2013), vários fatores influenciam a dinâmica de crescimento, como os acontecimentos cotidianos da vida da criança e a sua interação com as pessoas com quem ela convive.

Ainda segundo a autora, a criança possui necessidades básicas e busca satisfazê-las. Quando essa satisfação ocorre de maneira direta, a criança está ajustada. A situação de desajuste da criança é discutida por Axline quando a busca por satisfação é bloqueada de alguma maneira. Assim, a criança continua buscando satisfação, mesmo que em sofrimento. A satisfação encontrada pela criança na condição de desajuste seria distorcida e desencadeadora de sofrimento emocional. Verificamos que, apesar de Axline ter publicado sua obra no período de não diretividade da perspectiva rogeriana, ela antecipa algumas noções acerca do desenvolvimento da personalidade que Rogers apresentará posteriormente (Rogers, 1957/1994; Kinget \& Rogers, 1962/1977), tais como: o desenvolvimento do eu, a necessidade de consideração positiva de si, o desenvolvimento de um modo de avaliação condicional e o desacordo entre experiência e self (Brito \& Paiva, 2012).

A base da Ludoterapia de Axline é a de que a criança tem a capacidade de resolver satisfatoriamente seus problemas (Landreth, 2001/2012). A ludoterapeuta estabelece com a criança uma comunicação pela brincadeira e cria um espaço de expressão dos sentimentos acumulados e de ampliação de sua consciência. A ludoterapeuta não se apresenta como figura de autoridade ou como substituto dos pais. Ele está sempre alerta ao que a criança expressa de forma genuinamente interessada, o que se manifesta na terapia através de atitudes e não de técnicas (Axline, 1947/2013).

Apesar de apresentar a criança e a terapeuta em uma posição de maior atividade no desenvolvimento dos atendimentos, é conferido a essa segunda um papel diferenciado, já que, para Axline (1947/2013), o sucesso da psicoterapia é de inteira responsabilidade da terapeuta. Ela deve demonstrar segurança, evitando envolver-se emocionalmente com a criança, o que desvirtuaria a Ludoterapia. A terapeuta respeita a criança, não assumindo nem uma postura rígida, nem excessivamente flexível. Além disso, a terapeuta deve desenvolver seu atendimento a partir de princípios, o que reforça, em nossa compreensão, a maior responsabilidade da psicoterapeuta e uma desigualdade na participação em relação à criança.
Os oito princípios propostos por Axline (1947/2013), são: o desenvolvimento de um relacionamento com a criança, para que se estabeleça o rapport; a aceitação da criança como ela é; o estabelecimento do sentimento de permissividade na ludoterapia, para que a criança expresse livremente seus sentimentos; a terapeuta deve estar sempre alerta para identificar os sentimentos expressos pela criança e para refleti-los para ela; o respeito pela capacidade da criança de assumir a responsabilidade e resolver seus problemas; não dirigir as ações ou conversas da criança de forma alguma, deixando que ela indique o caminho; não abreviar a duração da terapia; o estabelecimento de limites mínimos necessários para o desenvolvimento da terapia. Assim como Rogers, Axline afirmava que a conduta dos pais, em muitos casos, poderia agravar os problemas da criança. No entanto, ela se diferenciou por não exigir que os pais iniciassem psicoterapia enquanto a criança estivesse em atendimento. Axline considerou a importância da participação dos pais no processo da criança (Stulmaker \& Jayne, 2017) e, ainda que não exigisse, não descartava que a psicoterapia da criança poderia ser otimizada caso o atendimento dos pais ocorresse simultaneamente (Axline, 1947/2013).

Em Ludoterapia - A dinâmica interior $d a$ infância, Axline avança em discussões apresentadas por Rogers (1942/2005). Mesmo denominando sua Ludoterapia de não-diretiva, a autora diferencia-se em seus posicionamentos, o que sugere, segundo Brito e Paiva (2012), uma transição entre a fase não-diretiva e a reflexiva da obra rogeriana. A compreensão sobre as implicações do processo terapêutico para a criança e seus pais difere das descritas por Rogers na fase não-diretiva. Compreendemos que Axline antecipa, ainda, algumas formulações publicadas posteriormente por seu mentor acerca da teoria da personalidade e das condições necessárias e suficientes para a psicoterapia, ainda que não avance, conforme apontado por Brito e Paiva (2012), para que a consideremos uma autora representativa da fase reflexiva (Segrera 2002; Moreira, 2010). Consideramos que a Ludoterapia proposta por Axline pode representar uma fase específica, dada a sua relevância no campo da psicoterapia com crianças (Baggerly, Ray \& Bratton, 2010; Landreth, 2001/2012; Bratton, Purswell \& Jayne, 2015; Glover \& Landreth, 2016). Propomos que essa fase seja denominada de "auto-diretiva" por se tratar de um termo, segundo a própria Axline (1947/2013), mais condizente com sua proposta interventiva.

\section{A Ludoterapia na Fase Reflexiva (1950-1957)}

Aluna de Rogers no Counseling Center em Chicago, Elaine Dorfman publicou capítulo sobre Ludoterapia no livro Terapia Centrada no Cliente, de 1951. Nessa obra, Rogers apresenta a teoria da 
personalidade e da Terapia Centrada no Cliente, bem como as implicações da teoria em contextos diversos, dentre eles a psicoterapia com crianças. Pouco citada na literatura sobre psicoterapia com crianças, Dorfman (1951/1992; 1958) discutiu sobre essa prática a partir da perspectiva rogeriana descrita na fase reflexiva e pesquisou sobre as mudanças de personalidade a partir da ludoterapia.

Para Dorfman, a base para a Ludoterapia é a capacidade do indivíduo para o crescimento, desenvolvimento e autodireção (Wilson \& Ryan, 2008). Sobre as atitudes da criança e a indicação de terapia, a autora lança um olhar para as suas formas de comportamento: "Por mais inadequados que possam ser, são a maneira dela [da criança] de resolver seus problemas" (Dorfman, 1951/1992, p. 273). Neste sentido, a psicoterapia pode contribuir diante das manifestações apresentadas pela criança, mas se o real problema não for cuidado, a contribuição seria apenas temporária, o que confere mais atenção à criança do que a quem a encaminha.

À medida que o trabalho com as crianças sem o contato com os pais - acontecia com êxito, Dorfman passou a questionar-se sobre como as crianças conseguiriam lidar com seus conflitos sem que seu ambiente mudasse (Dorfman, 1958; West, 2000). A autora verificou que as mudanças vivenciadas pela criança na Ludoterapia são inevitavelmente percebidas em seus contextos: "Uma vez que a criança é percebida de maneira diferente, a reação a ela também é diferente, e essa diferença no tratamento pode levá-la a mudar ainda mais" (Dorfman, 1951/1992, p. 274).

Para Dorfman (1951/1992), a criança deve ter papel ativo na ludoterapia e certas condições são comunicadas para que ela se sinta segura no ambiente terapêutico. As condições a serem comunicadas são: a atitude de respeito (quando a terapeuta cria uma relação de compreensão, aceitação e segurança); o fato de que a terapia pertence à criança (não cabendo à terapeuta exercer um papel de liderança); a atitude de aceitação pela criança, não tentando moldá-la em uma forma socialmente aprovada (West, 2000).

O setting terapêutico, para Dorfman, não deve ser pedagogizante, mas também não tem liberdade ilimitada. Os limites apresentados têm relação com o comportamento da criança. A expressão verbal dos sentimentos da criança é totalmente livre, ainda que não haja "permissão para que alguns sentimentos sejam expressos em ação" (Dorfman, 1951/1992, p. 294). Ou seja, determinados comportamentos, e não os sentimentos ligados àqueles, é que são limitados. Como esses sentimentos não são negados pela terapeuta, ela aprende a reconhecê-los, buscando formas de lidar com eles.

Os limites apresentados por Dorfman diferenciam-se dos de Axline: para esta, os limites situam a criança no campo da realidade; para aquela, os limites mantêm a aceitação da terapeuta.
West (2000) ressalta, com base na perspectiva de Dorfman, a importância da atitude de abertura da psicoterapeuta para o que a criança apresenta no desenvolvimento das sessões e enfatiza o potencial terapêutico dessa disponibilidade. Em Dorfman, os limites relacionam-se diretamente com a participação subjetiva da terapeuta na relação, característica da fase reflexiva (Segrera, 2002; Moreira, 2010).

Como consequência da psicoterapia, a criança passa a realizar a "transferência de comportamentos" da Ludoterapia para as situações cotidianas. Os limites, portanto, além de sustentarem a atitude de aceitação da terapeuta, têm potencial socializante, estruturam o espaço psicoterapêutico e conferem segurança, promovendo a redução da ansiedade da criança (Dorfman 1958).

No trabalho de Dorfman, apesar de a autora ter desenvolvido apenas dois trabalhos, ressaltamos a importância da manutenção da atitude de aceitação diante da criança, o que sinaliza o destaque da participação subjetiva da terapeuta no processo, algo não preconizado anteriormente nem por Rogers nem por Axline.

\section{A Ludoterapia na Fase Experiencial (1957 - 1970)}

A compreensão da psicoterapia como processo, o foco na experiência vivida, tanto do cliente como da terapeuta e a relação como promotora de crescimento, são características da fase experiencial (Segrera, 2002; Moreira, 2010; Melo et al., 2014) que são verificadas na obra de Clark Moustakas. Aluno de Axline, Moustakas foi influenciado diretamente por sua professora e também por Rogers (Moustakas, 1953; Blau, Bach, Scott, \& Rubin, 2013; Crenshaw \& Kenney-Noziska, 2014). Autor de diversas obras sobre atendimento infantil, Moustakas propôs a ênfase no desenvolvimento da interação entre a terapeuta e a criança e no estabelecimento de atitudes com a finalidade de aprofundamento da relação terapêutica (Crenshaw \& Kenney-Noziska, 2014).

A psicoterapia infantil, para Moustakas, é considerada uma relação de ajuda e uma experiência de crescimento, na qual um se disponibiliza para ajudar e o outro aceita ser ajudado. A relação terapêutica com a criança, através da brincadeira, representa um ato receptivo e não o posicionamento passivo de algum dos participantes (Moustakas, 1959; 2007). Compreendemos que esse posicionamento acerca da psicoterapia converge com a noção de relação de ajuda, proposta por Rogers (1961/2001), como uma experiência da relação intersubjetiva vivida pelo cliente e pelo terapeuta (Melo et al., 2014).

Para Moustakas (2007), a psicoterapia é uma relação que propicia à criança crescimento emocional e maior confiança em si mesma. A partir da presença do ludoterapeuta, vivenciando as atitudes de 
confiança, aceitação e respeito, ocorre a ênfase na expressividade e na clarificação dos sentimentos da criança. O autor considera que o desenvolvimento da relação ocorre a partir da noção de presença que é entendida como o comprometimento e a atenta escuta do que a criança expressa.

A participação dos pais e familiares no atendimento infantil possibilitava que eles pudessem aprender e aplicar os valores da ludoterapia com seus filhos. Antes de receber a criança para atendimento, ele atendia os pais. Após esse momento, atendia a criança por três ou quatro sessões, traçando um planejamento que poderia incluir sessões conjuntas com a família, mas com foco na criança atendida. Após as sessões conjuntas, os pais iniciariam um processo de "treinamento" e aprendizado sobre a conduta com a criança. Sinalizamos aqui as primeiras alusões ao estabelecimento de uma metodologia de atendimento infantil, elemento que será bastante discutido contemporaneamente (Moustakas, 1959; 2007).

Moustakas (2007) se afastou da proposta de Axline e desenvolveu um modelo de Ludoterapia fundamentadonarelação. Desde suas primeiras obras, Moustakas $(1953 ; 1959)$ aponta a influência de Otto Rank e Jesse Taft como base para enfatizar a relação com a criança mais do que a não-direção desenvolvida por sua mentora (Johnson, 2016). Todavia, apesar do afastamento de Axline, percebemos na obra de Moustakas uma manutenção da proximidade com as ideias de sua mentora, especialmente nas atitudes vivenciadas pela ludoterapeuta na relação com a criança. A confiança, a aceitação, o respeito, bem como a importância dos limites, são parte dos oito princípios de Axline (1947/2013) parecem se manter inalterados (Landreth, 2001/2012; Johnson, 2016).

$\mathrm{O}$ avanço em relação à proposta de Axline e a aproximação da perspectiva rogeriana na fase experiencial aparecem quando o autor sublinha a relevância da presença e da experiência da terapeuta na relação com a criança. Moustakas (1959; 2007) ressalta ainda a importância da experiência mútua e ativa da terapeuta e do cliente na relação como fundamentos para o desenvolvimento da psicoterapia.

Verificamos na obra de Moustakas convergências com aspectos da fase experiencial rogeriana, como a ênfase na qualidade da interação pessoa a pessoa, pelas noções de presença, de diálogo e de construção da relação. Moustakas é citado como importante autor da Ludoterapia Centrada na Criança por sua contribuição teórica e prática (Lin \& Bratton, 2014; Johnson, 2016).

\section{A Ludoterapia na Fase Pós- rogeriana (1987 - dias atuais)}

Os trabalhos contemporâneos em Abordagem Centrada na Pessoa têm como ponto de partida alguma das fases do pensamento rogeriano e o diálogo com outras perspectivas. Isso significa que essas novas teorias sofrem influências que as diferenciam epistemológica e metodologicamente da teoria de Rogers, assumindo uma identidade própria (Segrera, 2002; Moreira, 2010; Berh \& CorneliusWhite, 2008; Branco \& Cirino, 2017).

A Ludoterapia Centrada na Criança desenvolvida contemporaneamente também apresenta mudanças. Verificamos que os autores propõem desenvolvimentos a partir dos seguintes eixos: metodologia de atendimento, atitudes da psicoterapeuta e qualidade da relação. Apontaremos alguns trabalhos considerados relevantes em três contextos: europeu, norte-americano e brasileiro. A especificidade na escolha de contextos se deve ao reconhecimento da origem das fontes bibliográficas. Verificamos significativa produção sobre Ludoterapia desenvolvida nos Estados Unidos, especialmente com ênfase no método para o atendimento de crianças com necessidades específicas. Entretanto, é importante ressaltar que o desenvolvimento metodológico, reconhecido no contexto norte-americano, também se apresenta na Europa e no Brasil.

\section{Ludoterapia Centrada na Criança - Europa}

Considerado um dos autores contemporâneos da Ludoterapia (Schaefer \& Kaduson, 2006; Berh \& Cornelius-White, 2008; Bratton, Ray, Lindo \& Landreth, 2014), Goetze apresenta a Ludoterapia Centrada na Pessoa como um modelo preventivo, curativo e pós-curativo de atendimento, caracterizado por atitudes da terapeuta na interação e por atividades desenvolvidas com a criança (Goetze, 1994). Essa interação é processual e dinâmica, indo de um inicial distanciamento à uma posterior proximidade entre a criança e aterapeuta. Esse modelo de estágios é descritivo de um processo descrito como "natural" (p. 64) na Ludoterapia Centrada na Pessoa, mas também normativo, por prever diferentes ações da terapeuta do processo.

Os quatro estágios apresentados por Goetze (1994) são: não-pessoal - vinculação e reconhecimento entre cliente e terapeuta; nãodiretivo- caracterizado pelos oito estágios de Axline (1947/2013); centrado no cliente - integração de informações sobre a criança e desenvolvimento de técnicas com a finalidade de resolução de problemas; e estágio centrado na pessoa - maior participação da terapeuta e maior integração das experiências vividas na terapia com a vida real, considerada uma etapa de finalização da terapia.

Os elementos de estágio anteriores podem continuar em estágios posteriores. A comunicação com a criança é mantida com foco na sua capacidade de resolução de problemas (Döring, 2008). As técnicas utilizadas podem ser de outras perspectivas teóricas, como o Psicodrama ou a Gestalt-terapia 
(Goetze, 1994). Compreendemos, assim como Berh e Cornelius-White (2008), que o trabalho de Goetze busca o estabelecimento de etapas que sistematizem o desenvolvimento da Ludoterapia.

Ainda no contexto europeu, a proposta de Behr (2003), diferente de Goetze, discute uma extensão da Ludoterapia Centrada na Criança a partir da ênfase na imediaticidade da relação terapeuta-criança. A partir da brincadeira, ocorre o estabelecimento de um contato imediato, promotor da redução da incongruência da criança e que seriam pouco acessíveis pela comunicação verbal. Esse contato foi descrito por Behr como compromisso relacional chamado de ressonância interativa.

A noção de ressonância interativa, apresentada por Berh (2003), nos permite compreender uma aproximação com a dimensão de intersubjetividade, através da experiência da criança e da psicoterapeuta, na relação interpessoal psicoterápica. Na brincadeira com a criança, a psicoterapeuta responde à brincadeira tanto pelas palavras, como pelas ações. Isso possibilita à psicoterapeuta manter-se em não-diretividade e promover novas alternativas de experiências na relação. A ressonância interativa aprimora e modifica, segundo Behr (2003), a atitudede empatia, relacionando-se também com a autenticidade e com a aceitação. Estas atitudes representariam apenas parcialmente as intervenções com a criança. Já a ressonância teria uma ótica mais compreensiva e de aprofundamento da relação com a criança. Em reação ao que a criança faz, a terapeuta demonstra presença e envolvimento pessoal na relação (Berh \& Cornelius-White, 2008).

No atendimento, a partir dos padrões de interação apresentados pela criança, a terapeuta oferece ressonância, verbal e não verbal, à forma de agir da criança (Döring, 2008). Para tal, a terapeuta comunica verbalmente sua compreensão empática, atenta aos afetos da criança para espelhá-los de forma não literal, mas ativa no contato com a criança (Behr, 2003).

Percebemos que o trabalho de Goetze enfatiza a proposição de estágios para o processo terapêutico, assim como as diferentes ações e técnicas desenvolvidas na relação com a criança em psicoterapia. O entendimento da Ludoterapia em uma perspectiva preventiva/curativa/póscurativa chama a atenção para uma possível ênfase na dimensão de adoecimento. Além disso, o direcionamento das intervenções, pela característica normativa de sua proposta, busca a resolução de problemas apresentados pela criança. Os estágios e as ações a serem seguidos pela psicoterapeuta, de um maior afastamento a uma maior aproximação da criança, podem distanciar psicoterapeuta e criança daquilo que se apresenta na imediaticidade do encontro.

As pesquisas desenvolvidas por Behr, a partir da noção de ressonância interativa, apontam uma outra direção. Ao colocar a ênfase do trabalho psicoterapêutico na qualidade da interação entre terapeuta e criança, na imediaticidade de seu acontecimento, promovem uma compreensão de que o processo de mudança na criança está diretamente relacionada à qualidade do encontro. Aqui, Berh assume uma postura de atenção ao aprofundamento relacional a partir da forma como a criança se apresenta no atendimento psicoterapêutico.

\section{Ludoterapia Centrada na Criança-América do Norte}

No contexto estadunidense verificamos que os estudos apresentam aprimoramentos teóricometodológicos para o atendimento infantil. Além das reformulações, os autores ressaltam a discussão acerca da eficácia da Ludoterapia para o acompanhamento de problemáticas específicas (Baggerly, Ray \& Bratton, 2010; Lin \& Bratton, 2014; O'Connor, Schaefer \& Braverman, 2016), tal como desenvolvido por Rogers (1942/2005; 1951/1992). Para este tópico, discutimos os trabalhos de Landreth (2001/2012) e VanFleet, Sywulak e Sniscak (2010).

\section{A Ludoterapia Centrada na Criança de Garry Landreth}

Garry Landreth, um dos primeiros autores a influenciar contemporaneamente a Ludoterapia na Abordagem Centrada na Pessoa (Wilson \& Ryan, 2008), publicou em Play Therapy - The Art of Relationship uma proposição de ampliação da Ludoterapia Centrada na Criança, abordando a ênfase na qualidade da relação como promotor de mudança infantil (Lin \& Bratton, 2014). Esse livro trata da relação entre terapeuta e criança, considerando que, ao invés de desenvolver técnicas no atendimento, a ludoterapeuta deve demonstrar profundo compromisso com a criança e sua capacidade de crescimento, maturidade, resiliência e autodireção. Os comportamentos apresentados pela criança indicam a percepção dela sobre si mesma. Essa percepção provê a base para o funcionamento da criança em suas experiências (Shottelkorb, Swan, \& Ogawa, 2015).

Para Landreth (2001/2012), uma teoria da personalidade centrada na criança seria estruturada em três construtos: a Pessoa, representada por tudo o que a criança é: pensamentos, comportamentos, sentimentos e o seu ser físico; o Campo fenomenal, compreendido como tudo o que a criança experiencia, consciente ou inconscientemente; o Self apresentado pelo autor como uma diferenciação das experiências da criança, pelo contato com as pessoas-critério, com o meio e o campo fenomenal, mudando como resultado da contínua interação com o mundo.

A psicoterapia de Landreth, ainda que parta dos princípios da ludoterapia de Axline, refina e desenvolve essa perspectiva (Johnson, 2016). Landreth enumera as dimensões terapêuticas da relação psicoterápica: a crença na criança e 
o respeito aos seus limites; a aceitação da sua vontade; a escuta do interior da criança; o foco em suas necessidades; a liberdade da criança para determinar a direção a ser vivenciada; a oportunidade para que ela faça escolhas; a paciência com o processo (Landreth, 2001/2012; Shottelkorb, Swan, \& Ogawa, 2015; Glover \& Landreth, 2016). Com base nessa psicoterapia e no estabelecimento de uma relação intersubjetiva com a criança, os objetivos esperados são: a facilitação dos esforços da criança para enfrentamento e resolução dos atuais e futuros problemas que se apresentem; ajudar a criança no aprendizado de uma liberdade expressiva responsável; a aceitação de seus sentimentos e o controle responsável destes; a responsabilização por si mesma; a busca de criatividade (Landreth, 2001/2012; Glover \& Landreth, 2016).

A Ludoterapia proposta por Landreth (2016) traz como ênfase a qualidade da relação estabelecida como fundamento para o desenvolvimento do processo com a criança, pois "a ludoterapia centrada na criança é uma relação pessoal na qual o ludoterapeuta se dedica o mais completamente possível na imediaticidade da relação" (p. VII). Isso confere ao contato psicoterapêutico um aprimoramento do contato e da presença da psicoterapeuta.

\section{A Ludoterapia Centrada na Criança em VanFleet, Sywulak e Sniscak}

VanFleet et al. (2010) partem da percepção da ausência de uma metodologia de atendimento na teoria de Axline, pois a autora "não descreveu metodologias em detalhes em seus escritos" (p. 42). Partindo dessa ausência realizam uma leitura crítica dessa teoria propondo uma sistematização metodológica. Os princípios de Axline são revisitados pelos autores, com exceção do quinto e do oitavo que, em seguida, apresentam a adição de habilidades a serem manifestas pela ludoterapeuta.

Para que o estabelecimento do rapport, primeiro princípio, aconteça satisfatoriamente, o terapeuta deve estar atento empaticamente, em sintonia com as necessidades da criança. A aceitação da criança, segundo princípio, deve ser comunicada através das respostas empáticas. Isso mostra que o terapeuta está em sintonia com o que a criança apresenta (Le Vay, 2016).

No terceiro princípio, a permissividade da terapeuta deve ser expressa a partir do interesse genuíno, pela atitude não julgadora e empática. O quarto princípio passa a ser descrito por VanFleet et al. (2010) pela noção de empatia ou reflexo de sentimentos. Estas noções são descritas indistintamente, apresentando características da compreensão empática e da resposta reflexa (Brito \& Freire, 2014), apesar de estas não serem sinônimas.

Para que a criança mostre o caminho, sexto princípio, as perguntas devem ser evitadas. As declarações devem expressar empatia e compreensão do mundo da criança. Os questionamentos são considerados diretivos e podem causar mudanças na natureza da brincadeira, por solicitarem da criança uma explicação que não partiu dela (Ray, 2014).

Além da revisão dos princípios de Axline, VanFleet et al. (2010) propõem quatro habilidades que demonstram à criança que ela é compreendida em seus sentimentos, comportamentos e desejos. As habilidades são: a estruturação; a escuta empática; a brincadeira imaginária e os limites do setting terapêutico (VanFleet \& Tophan, 2016). A estruturação auxilia a criança na compreensão de que a relação com a ludoterapeuta é diferenciada das outras de sua vida, estabelecendo o clima necessário para a Ludoterapia. A escuta empática é apresentada como uma mistura da consideração positiva incondicional e da congruência, e comunica a aceitação, a compreensão e a sintonia do terapeuta com a criança (Ray, 2014; Stulmaker, Lertora \& Garza, 2015). As atitudes facilitadoras de empatia, consideração positiva incondicional e congruência, apesar de se entrelaçarem na escuta terapêutica, não foram postuladas como uma mistura. Dessa forma, a escuta empática apresentada por VanFleet et al. (2010) não nos é claramente compreensível.

A brincadeira imaginária, segundo VanFleet e Tophan (2016), é um tipo de encenação que envolve o mundo da imaginação da criança. A terapeuta desempenha um papel imaginário, seguindo o caminho dado pela criança. Ocorre via solicitação da terapeuta, com a participação deste. Quanto aos limites estabelecidos, estes seriam mínimos, como os de Axline, e objetivam preservar a atmosfera de segurança.

Os trabalhos descritos no contexto estadunidense apresentam objetivos diferentes para a Ludoterapia Centrada na Criança. O trabalho de Landreth (2001/2012) enfatiza em sua obra a qualidade da relação estabelecida entre terapeuta e criança. Há uma ênfase na postura de compromisso e respeito com a criança e não na busca de desenvolvimentos técnicos. O reconhecimento de como a criança se percebe e de como se relaciona com o mundo são o mote para as dimensões terapêuticas que se desenvolvem na situação do encontro, no aqui e agora, com a criança.

VanFleet et al. (2010) propõem uma metodologia de atendimento infantil, a partir da releitura de alguns princípios da obra de Axline. Verificamos que o foco do trabalho dos autores é a busca de uma sistematização e o desenvolvimento de uma metodologia de atendimento na Ludoterapia. Para tal, estabelecem uma compreensão particular das atitudes da psicoterapeuta na relação com a criança. Ainda que para Rogers (1957/1994) exista uma interdependência entre as atitudes, cada uma delas apresenta conceituações que as diferenciam. Não verificamos uma diferenciação clara das atitudes 
apresentadas nem de como estas, no formato como se apresentam no texto dos autores, contribuiriam para a qualidade da relação com a criança. O componente relacional na psicoterapia é citado pelos autores, mas não enfatizado.

\section{Ludoterapia Centrada na Criança - contexto brasileiro}

No Brasil, os trabalhos se apresentam em menor quantidade, quando comparamos com outros contextos (Brito \& Freire, 2014; Silva \& Barroso, 2017). O desenvolvimento da Abordagem Centrada na Pessoa no Brasil tem ocorrido em articulação com vertentes fenomenológicas e existenciais (Moreira, 2010; Vieira \& Pinheiro, 2013; Branco, Matos, Sampaio \& Amaral, 2017). Os trabalhos que discutem a Ludoterapia Centrada na Criança desenvolvida no Brasil, seguindo a mesma direção, tem se articulado com a fenomenologia (Campos \& Cury, 2009; Brito \& Freire, 2014), assim como enfatizam a qualidade da relação estabelecida entre psicoterapeuta e criança. $\mathrm{Na}$ discussão acerca do desenvolvimento da Ludoterapia em contexto brasileiro, esse artigo seguirá a direção da apresentação de estudos que propõem revisões mais breves do trabalho de Axline, sem maiores compromissos de aprofundamentos conceituais ou metodológicos; estudos que, inspirados pelo trabalho de Axline, apontam a relevância de seus princípios em contextos diversos; e estudos que, em diálogo com a perspectiva fenomenológica, propõem uma releitura da Ludoterapia Centrada na Criança.

Reconhecemos também a existência do desenvolvimento de trabalhos que discutem intervenções com crianças a partir da perspectiva de Axline, articuladas com o proposto por Rogers em momentos posteriores de sua teoria. Branco (2001) desenvolve um estudo de caso sobre a inserção da literatura como possibilidade interventiva na Ludoterapia Centrada na Criança. Nesse trabalho, discutiu a prática da Ludoterapia articulando as condições necessárias de Rogers, os princípios da ludoterapia de Axline e as atitudes de Landreth estas últimas discutidas no subtópico anterior. Essas perspectivas foram apresentadas conjuntamente e a discussão acerca dos diferentes períodos nos quais essas propostas se apresentavam foi pouco explorada no estudo.

Garcia (2002) apresenta diretrizes de um Ludoterapia Centrada na Criança e organiza seu estudo como uma revisão descritiva da obra Ludoterapia - a dinâmica interior da infância de Axline, apresentando seus princípios e outros elementos discutidos pela autora clássica para o desenvolvimento de uma Ludoterapia na Abordagem Centrada na Pessoa. Ressaltamos uma ampliação de seu trabalho em relação ao proposto por Axline: a construção de uma breve discussão acerca dos elementos metodológicos das primeiras sessões com a família e com a criança. Contudo, a autora não aprofunda na temática e nem na articulação com a psicoterapia.

Campos e Cury (2009), em pesquisa sobre atenção psicológica em uma creche, e Giaxa, Tavares, Oliveira, Eying e Burda (2019), em estudo sobre a dimensão terapêutica dos jogos no processo de hospitalização de crianças apresentam, em perspectivas diferentes, como o trabalho clássico de Axline tem relevância na discussão e compreensão do trabalho com crianças em contextos que extrapolam a prática da Ludoterapia como prática psicológica clínica.

Brito e Freire (2014) propõem uma releitura da Ludoterapia Centrada na Criança, a partir da fenomenologia filosófica de Emmanuel Lévinas, discussão já realizada anteriormente entre essa fenomenologia e a Abordagem Centrada na Pessoa (Vieira \& Freire, 2006; Vieira \& Pinheiro; 2013). As atitudes e habilidades do terapeuta são revistas para que haja espaço para a responsabilidade (habilidade de resposta) ao chamado da Outro e para a experiência traumática e desalojadora na Ludoterapia. Estes elementos são promotores de uma relação facilitadora e promotora de crescimento.

Compreender um possível espaço para a alteridade na Ludoterapia Centrada na Criança exige da psicoterapeuta o reconhecimento de suas ideias de infância e o posterior afastamento dessas compreensões. Assim, é possível perceber o quanto há de abertura à alteridade ou do quanto encaixa-se a criança em critérios familiares. O reconhecimento e afastamento das preconcepções promove abertura e disponibilidade à imprevisibilidade e à diferença, possibilitando a afetação da psicoterapeuta e da criança na relação (Brito, 2012; Brito \& Freire, 2014).

Essa abertura exige da ludoterapeuta a saída de uma comunicação que "encaixe" a fala da criança nos parâmetros do adulto para um movimento contrário, no qual a terapeuta aproxima-se da criança como ela se apresenta, o que nos direciona para uma outra compreensão acerca da infância e de sua condição de estrangeira (Frota, 2018). O estabelecimento de comunicação se dá a partir de vias de "estranhamento", que exigem da psicóloga reconfigurações, 'traumatismos'. O encontro com a criança é a lida com a alteridade em sua absoluta radicalidade (Brito \& Freire, 2014).

Na ocorrência desse encontro na ludoterapia, como descrito por Brito (2012) é necessário colocarse em diaconia (serviço), pela disponibilidade organísmica do terapeuta. Essa atitude diz respeito à qualidade de escuta, à resposta verbal, mas também à disponibilidade física. Nosso organismo torna-se um instrumento terapêutico (Andrade \& Cavalcante Jr., 2008; Brito \& Freire, 2014) que passa a fazer parte da brincadeira, para responder à criança em suas experiências lúdicas.

Para compreender essa forma de contato 
na Ludoterapia Centrada na Criança, os estudos propõem o reconhecimento da influência da obra de Axline (1947/2013), mas também a releitura dos princípios da autora, articulando esses princípios com as atitudes facilitadoras (Rogers, 1957/1994) e o entendimento da relação intersubjetiva como promotora de mudança e como encontro existencial, característicos da fase experiencial (Brito, 2012; Brito \& Freire, 2014).

Os princípios de Axline (1947/2013) abordados e rediscutidos por Brito e Freire (2014) são: o rapport, que passa a ser entendido como disponibilidade da psicoterapeuta para a revelação da criança e de sua alteridade; a aceitação, estendida para a noção de consideração positiva incondicional, via descentramento do mundo adulto; do sentimento de permissividade da terapeutapara a ideia de psicoterapia como encontro intersubjetivo; o reflexo dos sentimentos, expandido para a compreensão empática como atitude de "não saber"; o respeito pela criança como indivíduo estende-se para a compreensão da criança como ser individual e relacional; da confiança na criança para a psicoterapia como intersubjetividade e via de responsabilidade (habilidade de resposta); da impossibilidade de apressar a psicoterapia para a compreensão desse processo como experiência de desenvolvimento de autonomia e de habilidades relacionais; dos limites, que representam a alteridade da terapeuta para a criança.

No contexto brasileiro, o desenvolvimento da Ludoterapia Centrada na Criança enfatiza a qualidade da relação terapêutica e as atitudes da psicoterapeuta na relação com a criança. A psicoterapeuta não é a principal ou única responsável pela manutenção da psicoterapia. Na psicoterapia, a abertura à alteridade e à diferença devem ocorrer tanto na criança quanto na terapeuta de forma intersubjetiva (Garcia, 2002; Schmidt, 2013; Brito \& Freire, 2014; Giaxa, Tavares, Oliveira, Eying \& Burda, 2019).

\section{Considerações Finais}

Visando contribuir com uma discussão histórica acerca da Ludoterapia Centrada na Criança, apontamos, no decorrer deste artigo, a necessidade do reconhecimento das mudanças ocorridas nessa modalidade de intervenção com crianças, bem como da proposição de fases de desenvolvimento da psicoterapia infantil na Abordagem Centrada na Pessoa.

Nessa medida foram apresentadas e discutidas as características da Ludoterapia, nome convencionado para a psicoterapia com crianças na Abordagem Centrada na Pessoa, a partir das fases do pensamento rogeriano. Esse percurso histórico iniciou nos fundamentos iniciais do atendimento com crianças ACP, seguido do reconhecimento da Ludoterapia nas diversas fases, a saber: a fase não- diretiva, que em nossa perspectiva se desmembra em uma ludoterapia não-diretiva em Rogers e em uma ludoterapia auto-diretiva em Axline; a fase reflexiva, cujo nome de Dorfman se destaca e sua construção acerca da participação intersubjetiva do psicoterapeuta; a fase experiencial, na qual ressaltamos o trabalho de Moustakas e a relevância da noção de presença no encontro com a criança; e a fase pós-rogeriana, que se caracteriza pela diversidade de perspectivas e de desenvolvimento metodológico para a psicoterapia com crianças.

Concluímos que existe grande diversidade na teoria e prática da Ludoterapia Centrada na Criança. Ressaltamos inicialmente o trabalho de Rogers com crianças como parte relevante do desenvolvimento histórico da Ludoterapia Centrada na Criança, mas que não costuma ser abordado ou mesmo reconhecido em outros estudos. Contemporaneamente, apesar de os trabalhos nessa intervenção apontarem como principal fundamento teórico a ludoterapia nãodiretiva de Axline, as pesquisas têm apresentado ampliações e desenvolvimentos que se diferenciam do proposto pela autora. Destacamos aqui desde os trabalhos de Moustakas (que amplia a noção de não-diretividade de Axline através da ênfase na construção relacional intersubjetiva), assim como os trabalhos desenvolvidos por autores contemporâneos. Nestes, ora há proposições para um desenvolvimento metodológico, como verificamos em Goetze, VanFleet, Sywulak e Sniscak, ora para o aprimoramento da psicoterapia, através do aprofundamento da relação intersubjetiva estabelecida, como proposto por Berh, Landreth, Brito e Freire.

Ressaltamos que a delimitação do atendimento infantil na Abordagem Centrada na Pessoa em fases possibilita uma percepção mais clara das mudanças e aprimoramentos em relação aos trabalhos pioneiros de Rogers e Axline. Trata-se de um campo fecundo em desenvolvimento que tanto mantém as bases históricas da Abordagem Centrada na Pessoa quanto incorpora novos movimentos, sempre tendo como eixo a dimensão relacional e a tendência ao crescimento como motor de todo e qualquer processo.

\section{Referências}

Andrade, A. C. \& Cavalcante Júnior, F. S. (2008). A expressão da tendência formativa na Abordagem Centrada na Pessoa. Em Cavalcante Júnior, F. S., \& Sousa, A. F. Humanismo de Funcionamento Pleno: Tendência Formativa na Abordagem Centrada na Pessoa - ACP (pp. 157-190). Campinas: Alínea.

Axline, V. M. (2013). Play Therapy - The Inner Dinamics of Childhood. Canada: Hesperides Press. (Originalmente publicado em 1947). 
Baggerly, J. N., Ray, D. \& Bratton, S. C. (2010). Child-Centered Play Therapy Research: An Evidence Base for Effective Practice. New Jersey: John Wiley \& Sons.

Behr, M. (2003). Interactive Resonance in Work with Children and Adolescents: A theory-based of interpersonal relationship through play and the use of toys. Person-Centered and Experiential Psychotherapies, 2(2), 89-103.

Behr, M. \& Cornelius-White, J. (2008). Facilitanting Young People's Development: International perspectives on person-centred theory and practice. Gateshad: PPCS Books

Blau, D., Bach, L., Scott, M., \& Rubin, S. (2013). Clark Moustakas (1923-2012): Scholar, Teacher, Colleague and Friend. The Humanistic Psychologist, 41(1), 97-99.

Branco, T. M. C. (2001). Histórias Infantis na Ludoterapia Centrada na Criança (Dissertação de Mestrado). Pontifícia Universidade Católica de Campinas - PUC-CAMPINAS, Campinas.

Branco, P. C. C. (2010). A noção de organismo no fieri teórico de Carl Rogers: uma investigação epistemológica (Dissertação de Mestrado). Universidade Federal do Ceará, Fortaleza.

Branco, P. C. C. \& Cirino, S. D. (2017). Fenomenologia nas obras de Carl Rogers: apontamentos para o cenário brasileiro. Revista de Psicologia, $8(2), 44-52$.

Branco, P. C. C., Vieira, E. M., Cirino, S. D., \& Moreira, J. O. (2016). Influência da Psicanálise neofreudiana na Psicoterapia de Carl Rogers. Contextos Clínicos, 9(2), 279-289.

Branco, P. C. C., Matos, G. N., Sampaio, A. G. S., \& Amaral, B. R. (2017). Formação do Psicólogo Humanista: Revisão Sistemática. Perspectivas em Psicologia, 21(1), 73-92.

Bratton, S. C., Ray, D. C., Lindo, N. A., \& Landreth, G. (2014). Terapia de Juego Centrada en el Niño (TJCN): teoria, investigación y práctica. Em Segrera, A. Cornelius-White, J. H. D., Berh, M., \& Lombardi, S. Consultorías y psicoterapias centrada en la persona y experienciales: Fundamentos, perspectivas y aplicaciones (pp. 249270). Buenos Aires: Gran Aldea.

Bratton, S. C., Purswell, K., \& Jayne, K. (2015). Play Therapy: A Child-Centered Approach. Em Prout, H. T., \& Fedewa, A. L. Counselling and Psychotherapy with Children and Adolescents: Theory and Practice for School and Clinical Settings (pp. 91-114). New Jersey: Wiley and Sons.
Brito, R. A. C. (2012). A criança como Outro: uma leitura ética da Ludoterapia Centrada na Criança (Dissertação de Mestrado). Universidade Federal do Ceará, Fortaleza.

Brito, R. A. C., \& Paiva V. M. B. (2012). Psicoterapia de Rogers e Ludoterapia de Axline: Convergências e Divergências.Revista do NUFEN, 4(1), 102-114.

Brito, R. A. C., \& Freire, J. C. (2014). Ludoterapia Centrada na Criança - Uma Leitura a partir da Ética de Emmanuel Lévinas. Revista da Abordagem Gestáltica - Phenomenological Studies, 20(1), 118-127.

Campos, A., \& Cury, V. (2009). Atenção psicológica clínica: encontros terapêuticos com crianças em uma creche. Paidéia, 19(42), 115-121.

Crenshaw, D. A., \& Kenney-Noziska, S. (2014). Therapeutic Presence in Play Therapy. International Journal of Play Therapy, 23(1), 31-43.

Dorfman, E. (1958). Personality Outcomes of Client Centered Play Therapy. Psychological Monographs: General and Applied, 72(3), 1-22.

Dorfman, E. (1992). Ludoterapia. Em Rogers, C. R. Terapia Centrada no Cliente. São Paulo: Martins Fontes (Originalmente publicado em 1951)

Döring, E. (2008). What Happens in Child-Centered Play Therapy. Em Behr, M., \& Cornelius-White, J. Facilitanting Young People's Development: International perspectives on person-centred theory and practice (pp. 40-51). Gateshad: PPCS Books.

Elias, C. S. R., Silva, L. A., Martins, M. T. S. L., Ramos, N. A. P., Souza, M. G. G., \& Hipólito, R. L. (2012). Quando chega o fim? Uma revisão narrativa sobre terminalidade do período escolar para alunos deficientes mentais. Revista Eletrônica Saúde Mental Álcool e Drogas, 8(1), 48-53.

Frota, A. M. M. C. (2018). Infância, filosofia da educação e fenomenologia: aproximações necessárias. Revista da Abordagem Gestáltica - Phenomenological Studies, 24(1), 89-90.

Gadamer, H-G. (2008). Verdade e Método: Traços fundamentais para uma hermenêutica filosófica. Petrópolis: Vozes.

Garcia, S. S. (2002). Diretrizes da ludoterapia na abordagem centrada na pessoa. Em Gobbi, S. L. et al. Vocabulário e noções básicas da abordagem centrada na pessoa (pp. 183-201). São Paulo: Vetor. 
Giaxa, A. C. M., Tavares, E. M., Oliveira, T. P., Eying, J., \& Burda, T. A. M. (2019). A utilização do jogo como recurso terapêutico no processo de hospitalização da criança. Revista SBPH, 22(1), 280-305.

Glover, G., \& Landreth, G. (2016). Child-Centered Play Therapy. Em O’Connor, K. J., Schaefer, C. E., \& Braverman, L. D. Handbook of Play Therapy (pp. 93-118). New Jersey: Wiley \& Sons.

Goetze, H. (1994). Processes in Person-Centered Play Therapy. Em J. Hellendoorn, R. van der Kooji, \& B. Sutton-Smith (Eds.), Play and Intervention (pp. 63-76). Albany: State University of New York Press.

Hart, J. T., \& Tomlinson, T. M. (1970). New directions in client-centered therapy. Boston: Houghton Miffin.

Hillman, H. (2018). Child-Centered Play Therapy as an intervention for children with autism: A literature review. International Journal of Play Therapy, 27(4), 198-204.

Huizinga, J. (1984). Developments in life and work of Carl Ramson Rogers. Em Segrera, A. Proceedings of the First International Forum on the Person Centered Approach. Mexico: Universidad Iberoamericana.

Johnson, J. L. (2016). The History of Play Therapy. Em O’Connor K. J., Schaeffer, C. E., \& Braverman, L. D. Handbook of Play Therapy (pp. 1734). New Jersey: Wiley \& Sons.

Kinget, G. M., \& Rogers, C. R. (1977). Psicoterapia e Relações Humanas, Vol. I. Belo Horizonte: Interlivros. (Originalmente publicado em 1962).

Kirschenbaum, H. (2007). The life and work of Carl Rogers. England: PCCS Books.

Landreth, G. L. (2012). Play Therapy: The Art of the Relationship. New York, NY: Routledge Press. (Originalmente publicado em 2001).

Le Vay, D. (2016). To be or not to be? The therapeutic use of self within child-centred play therapy. Em Le Vay, D., \& Cuschieri, E. Challenges in theory and practice of Play Therapy (pp. 1-17). New York: Routledge.

Lin, Y-W., \& Bratton, S. C. (2014). A Meta-Analytic Review of Child-Centered Play Therapy Approaches. Journal of Counseling \& Development, 93(1), 45-58.

Melo, A. K. S., Lima, R. P., \& Moreira, V. (2014). Construção da Noção de Experiência ao Longo do Pensamento de Carl Rogers. Revista do NUFEN, 7(1), 4-31.
Moreira, V. (2010). Revisitando as Fases da Abordagem Centrada na Pessoa. Estudos de Psicologia (Campinas), 27(4), 537-544.

Moustakas, C. E. (1953). Children in Play Therapy: A key to understand normal and disturbed emotions. New York: McGraw-Hill Book Company

Moustakas, C. E. (1959). Psychotherapy with children: the living relationship. New York: Ballantine Books.

Moustakas, C. E. (2007). Relationship Play Therapy. Maryland: Jason Aronson Book.

O’Connor K. J., Schaeffer, C. E., \& Braverman, L. D. (2016). Handbook of Play Therapy. New Jersey: Wiley \& Sons.

Perryman, K. L., \& Bowers, L. (2018). Turning the Focus to Behavioral, Emotional and Social Well-Being: The Impact of Child-Centered Play Therapy. International Journal of Play Therapy, 27(4), 227-241.

Puente, M. (1970). Carl Rogers: de la psychothérapie à l'enseignement. Paris: EPI Editeurs.

Ray, D. (2014). A Way of Being in Playroom: Experience Expression Congruence Model. International Journal of Play Therapy, 23(1), 18-30.

Rogers, C. R. (1978). O tratamento clínico da criança problema. São Paulo: Martins Fontes. (Originalmente publicado em 1939).

Rogers, C. R. (1992). Terapia Centrada no Cliente. São Paulo: Martins Fontes. (Originalmente publicado em 1951)

Rogers, C. R. (1994). As condições necessárias e suficientes para a mudança terapêutica de personalidade. Em Wood. J. K. (Org.), Abordagem Centrada na Pessoa (pp. 155-177). Vitória: Ed. Fundação Ceciliano Abel de Almeida (Originalmente publicado em 1957).

Rogers, C. R. (2001). Torna-se Pessoa. São Paulo: Martins Fontes. (Originalmente publicado em 1961).

Rogers, C. R. (2005). Psicoterapia e Consulta psicológica. São Paulo: Martins Fontes. (Originalmente publicado em 1942).

Rogers, C. R. (2005). Um Jeito de Ser. São Paulo: EPU. (Originalmente publicado em 1980).

Schaefer, C. E., \& Kaduson, H. D. (2006). Contemporary Play Therapy: Theory, Research and Practice. New York: Guilford Press. 
Segrera, A. (2002). El enfoque centrado en la persona: reflexiones en el centenario de su fundador Carl Rogers. Miscelánea Comillas, 60(117), 399-419.

Shottelkorb, A. A., Swan, K. L., \& Ogawa, Y.(2015). Parent Consultation in Child-Centered Play Therapy: A Model for Research and Practice. International Journal of Play Therapy, 24(4), 221-233.

Silva, F. K. U., \& Barroso, A. C. (2017). Contribuição da Ludoterapia no Autismo Infantil. Saber $\mathrm{Hu}$ mano, 7(11), 210-224.

Sollod, R. (1978). Carl Rogers and the origins of client-centered therapy. Professional Psychology, 9(1), 93-104.

Stake, R. E. (2011). Pesquisa qualitativa: estudando como as coisas funcionam. Porto Alegre: Penso.

Stulmaker, H. L., Lertora, I., \& Garza, Y. (2015). Facilitating Empathic Perspective Taking in Beginning Child-Centered Play Therapists: The Role of Supervision. International Journal of Play Therapy, 24(4), 177-189.

Stulmaker, H. L., \& Jayne, K. M. (2017). Child-Centered Play Therapy Parent Consultation Model: Clinical Implementation and Implications. Journal of Child and Adolescent Counseling, 4(1), 3-19.

Telles, T. C. B., Boris, G. J. B., \& Moreira, V. (2014). O Conceito de Tendência Atualizante na Prática Clínica Contemporânea de Psicoterapeutas Humanistas. Revista da Abordagem Gestáltica - Phenomenological Studies, 20(1), 13-20.

VanFleet, R., Sywulak, A. E., \& Sniscak, C. C. (2010). Child Centered Play Therapy. New York, NY: The Guilford Press.

VanFleet, R., \& Tophan, G. L. (2016). Filial Therapy. Em McMahon, L. (Ed.). Handbook of play therapy and therapeutic play (pp. 135-165). New York: Routledge.

Vasconcelos, T. P. (2008) Concede-se Morte, Suscita-se Vida: um Golpe Formativo em um Grupo de Ludoterapia. Em Cavalcante Júnior, F. S., \& Sousa, A. F. Humanismo de Funcionamento Pleno: Tendência Formativa na Abordagem Centrada na Pessoa - ACP (pp. 145-156). Campinas: Alínea.

Vieira, E. M., \& Freire, J. C. (2006). Alteridade e Psicologia Humanista: uma leitura ética da abordagem centrada na pessoa. Estudos de Psicologia (Campinas), 23(4), 425-432.
Vieira, E. M., \& Pinheiro, F. P. H. A. (2013). Person centered psychotherapy: an encounter with oneself or a confrontation with the other? Estudos de Psicologia (Campinas), 30(2), 231-238.

West, J. (2000). Terapia de Juego Centrada en el Niño. Bogotá: Editorial el Manual Moderno.

Wilson, K., \& Ryan, V. (2008). Play Therapy: A Non-Directive Approach for Children and Adolescents. Edimburgh: Elsevier.

Wood. J. K. (1983). Terapia de Grupo Centrada na Pessoa. Em Rogers, C. R., Wood, J. K., O'Hara, M. M. \& Fonseca, A. Em busca de vida: da terapia centrada no cliente à abordagem centrada na pessoa (pp. 45-90). São Paulo: Summus.

Rosa Angela Cortez de Brito. Universidade de Fortaleza, Curso de Psicologia, Doutoranda do Programa de Pós-Graduação em Psicologia. Av. Washington Soares, 1321, Sl. N13, 60811-905, Fortaleza, Ceará, Brasil. Tel: (85) 988871054. Correspondência para: rosa@unifor.br.

José Célio Freire. Universidade Federal do Ceará.

Lucas Guimarães Bloc. Universidade de Fortaleza.

Virginia de Saboia Moreira Cavalcanti. Universidade de Fortaleza.

Recebido em 31.03.2020 Primeira decisão editorial 20.05.2020 Aceito em 11.01.2021 